\title{
Success of torsional correction surgery after failed surgeries for patellofemoral pain and instability
}

\author{
Peter M. Stevens • Jeremy M. Gililland • \\ Lucas A. Anderson • Jennifer B. Mickelson • \\ Jenifer Nielson · Joshua W. Klatt
}

Received: 23 February 2013/ Accepted: 29 November 2013/Published online: 15 December 2013

(C) The Author(s) 2013. This article is published with open access at Springerlink.com

\begin{abstract}
Torsional deformities of the femur and/or tibia often go unrecognized in adolescents and adults who present with anterior knee pain, and patellar maltracking or instability. While open and arthroscopic surgical techniques have evolved to address these problems, unrecognized torsion may compromise the outcomes of these procedures. We collected a group of 16 consecutive patients (23 knees), with mean age of 17 , who had undergone knee surgery before torsion was recognized and subsequently treated by means of rotational osteotomy of the tibia and/or femur. By follow-up questionnaire, we sought to determine the role of rotational correction at mean 59-month follow-up. We reasoned that, by correcting torsional alignment, we might be able to optimize longterm outcomes and avert repeated knee surgery. Knee pain was significantly improved after torsional treatment (mean 8.6 pre-op vs. 3.3 post-op, $p<0.001$ ), while $70 \%$ of patients did have some continued knee pain postoperatively. Only $43 \%$ of patients had continued patellar instability, and $57 \%$ could trust their knee after surgery. Activity level remained the same or increased in $78 \%$ of patients after torsional treatment. Excluding planned rod removal, subsequent knee surgery for continued anterior knee pain was undertaken on only 3 knees in 2 patients. We believe that malrotation of the lower limb not only raises the propensity for anterior knee symptoms, but is also a under-recognized etiology in the failure of surgeries for anterior knee pain and patellar instability. Addressing
\end{abstract}

P. M. Stevens $(\varangle) \cdot$ J. M. Gililland · L. A. Anderson ·

J. B. Mickelson - J. Nielson · J. W. Klatt

Department of Orthopaedic, Primary Children's Hospital,

University of Utah School of Medicine, 100 Mario Capecchi

Drive Suite 4550, Salt Lake City, UT 84113, USA

e-mail: peter.stevens@hsc.utah.edu; peter.stevens@mac.com rotational abnormalities in the index surgery yields better clinical outcomes than osteotomies performed after other prior knee surgeries.

Keywords Pan genu torsion - Miserable malalignment . Tibial torsion - Femoral anteversion · Osteotomy

\section{Introduction}

Anterior knee pain and patellofemoral instability are common causes of knee problems in both adolescents and adults. Theories as to the etiology of the above have included quadriceps muscle imbalance, retinacular/soft-tissue tightness, elevated "Q-angles," pathomorphology of the trochlea, and abnormal extensor complex (patella alta/baja) [1-3]. However, torsional deformities of the femur and/or tibia often go unrecognized in both adolescents and adults who present with anterior knee pain, and patellar maltracking and/or instability. While arthroscopic [4] and open [5, 6] surgical techniques have evolved to address the latter problems, the results may be compromised by underlying torsion. Failure to recognize and address the transverse plane deformities may prove disappointing to the patient and surgeon alike.

We hypothesized that there would be a satisfactory clinical improvement after rotational osteotomies of the tibia and/or femur in knees with unrecognized torsional abnormalities and ongoing knee problems after prior knee surgery for anterior knee pain or patellofemoral instability.

\section{Materials and methods}

This study was a retrospective review on a group of 18 patients who all had developed anterior knee pain and/or 
instability before skeletal maturity and had all undergone one or more prior knee surgeries before presenting to our clinic where torsional abnormalities were recognized (Fig. 1). Torsional deformities were treated by a single surgeon in our institution (PMS) by means of rotational osteotomy of the tibia and/or femur between May 1998 and November 2010.

We contacted these patients with a custom outcome questionnaire and 17 of 18 returned the questionnaire (Fig. 2). One of the 17 returned questionnaires contained incomplete data and was excluded. We therefore reviewed the records of 16 participating patients with 23 operated knees with mean 59-month follow-up (range 11-145 months). This group consisted of 13 females and 3 males. The mean age at the time of index rotational osteotomy was 17 , with a range of 9-30 years of age. The average number of prior knee surgeries per patient was 2 with a range of $1-4$ (Table 1). A patient case example is included in Fig. 3.

Prior to osteotomy, each patient had a "gunsight" rotational CT scan or MRI to document the degree of excessive torsion and determine whether to cut the femur,
Fig. 1 Torsion outcomes questionnaire
Patient's Name:

\section{Torsion Outcomes Questionnaire}

Date of Birth:

Please Circle your responses and fill-in an answer if necessary.

\section{1.) Do you continue to have knee pain/problems?}

$$
\begin{aligned}
& \text { Right Knee: Yes No } \\
& \text { If Yes: On a scale from 1-10 how severe is the pain? } \\
& \text { ( } 1 \text { being minimal and } 10 \text { extremely painful) } \\
& \text { Left Knee: Yes No } \\
& \text { If Yes: On a scale from 1-10 how severe is the pain? } \\
& \text { (1 being minimal and } 10 \text { extremely painful) }
\end{aligned}
$$

What was your pain on a scale from 1-10 before the osteotomy surgery?

$$
\text { Right: Left: }
$$

2.) Have you had any patellar instability since surgery?

$\begin{array}{lll}\text { Right Knee: } & \text { Yes } & \text { No } \\ \text { Left Knee: } & \text { Yes } & \text { No }\end{array}$

3.) Do you "trust" your knee? (Do you feel the knee is reliable and consistent?)

Right Knee: Yes No

Left Knee: Yes No

4.) Have you changed your lifestyle/activities?

$\begin{array}{ll}\text { Increased Activity Level } & \text { (More than before osteotomy surgery) } \\ \text { Same Activity Level } & \text { (No change from before osteotomy surgery) } \\ \text { Decreased Activity Level } & \text { (Less than before osteotomy surgery) }\end{array}$

5.) Have you had knee surgery since your osteotomy/hardware removal?

Yes No

If yes, what did you have done?

Thank you for choosing to participate by providing us with this information! 
Fig. 2 Bar graph illustrating types and number of prior failed surgeries before rotational malalignment addressed
Surgical Procedures Prior to Torsional Treatment

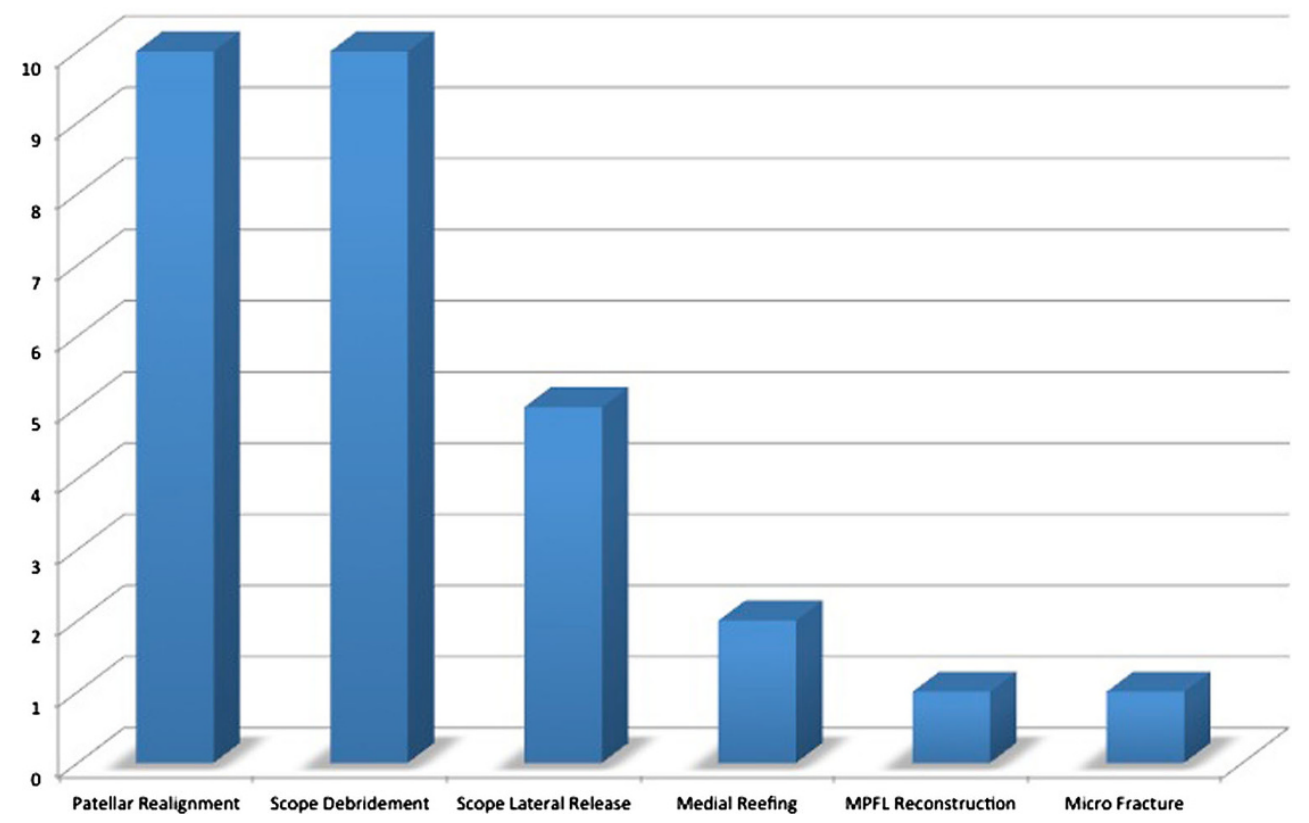

tibia, or both [2, 7, 8] (Fig. 3). Surgical interventions included femoral rotational osteotomy to correct anteversion, tibial rotational osteotomies to address outward tibial torsion, and simultaneous ipsilateral tibial and femoral osteotomies to address pan genu torsion (Tables 1 and 2). Surgical correction of the contralateral extremity was undertaken within 3 months in seven patients with bilateral symptoms.

The femoral osteotomies were performed percutaneously, at the mid-shaft level and stabilized with an antegrade, locked, intramedullary rod, using a transtrochanteric (not piriformis) entry point [9]. In skeletally mature patients, all tibial osteotomies were mid-shaft, leaving the fibula intact, and secured with an intramedullary rod [10]. If the patellar retinaculum was tight, as judged by a negative patellar inversion angle, then a lateral retinacular release was undertaken at the same sitting. The intramedullary rods were removed approximately 1 year after the osteotomies.

For the patients who were skeletally immature, the open proximal physis precluded the safe use of an intramedullary rod in the tibia. Instead, a supra-malleolar osteotomy of the tibia was performed and stabilized with two smooth, crossed Steinman pins, leaving the fibula undisturbed for stability and support [11]. Following 4 weeks in a belowthe-knee cast, the pins were removed in the clinic and the patients permitted weight bearing in a walking boot for an additional month.

Clinical outcomes were evaluated by a custom questionnaire (Fig. 1). This questionnaire asked about preoperative and postoperative pain levels, recurrent patellar instability, ability to "trust" the knee, postoperative activity levels, and any subsequent knee surgeries after the rotational osteotomy and hardware removal.

Data were analyzed by an independent statistician using commercially available software (STATA Version 11, College Station, TX). Student's t test was used for comparing the continuous variables of preoperative and postoperative pain.

\section{Results}

Knee pain on a scale of 1-10 was significantly improved after torsional treatment (mean 8.6 pre-op vs. 3.3 post-op, $p<0.001$ ), while $70 \%$ of patients did have some continued knee pain postoperatively (Table 3 ). Ten of 23 knees had continued patellar instability after torsional treatment, and 13 of 23 knees were "trusted" by the patient of after surgery. Activity level was improved after 15 of 23 of these cases, remained the same after in 3 knees, and was decreased after in 5 knees.

Our complication rate in this series was $8.7 \%$ with 2 complications encountered. The first complication was a femoral nonunion treated successfully with exchange reamed nailing. The second complication was a peroneal nerve irritation by a loose proximal tibial interlocking screw. This was treated successfully with hardware removal and peroneal neurolysis. Excluding planned rod removal, subsequent knee surgery for continued anterior knee pain was undertaken on three knees in two patients. One patient underwent bilateral guided growth to address 


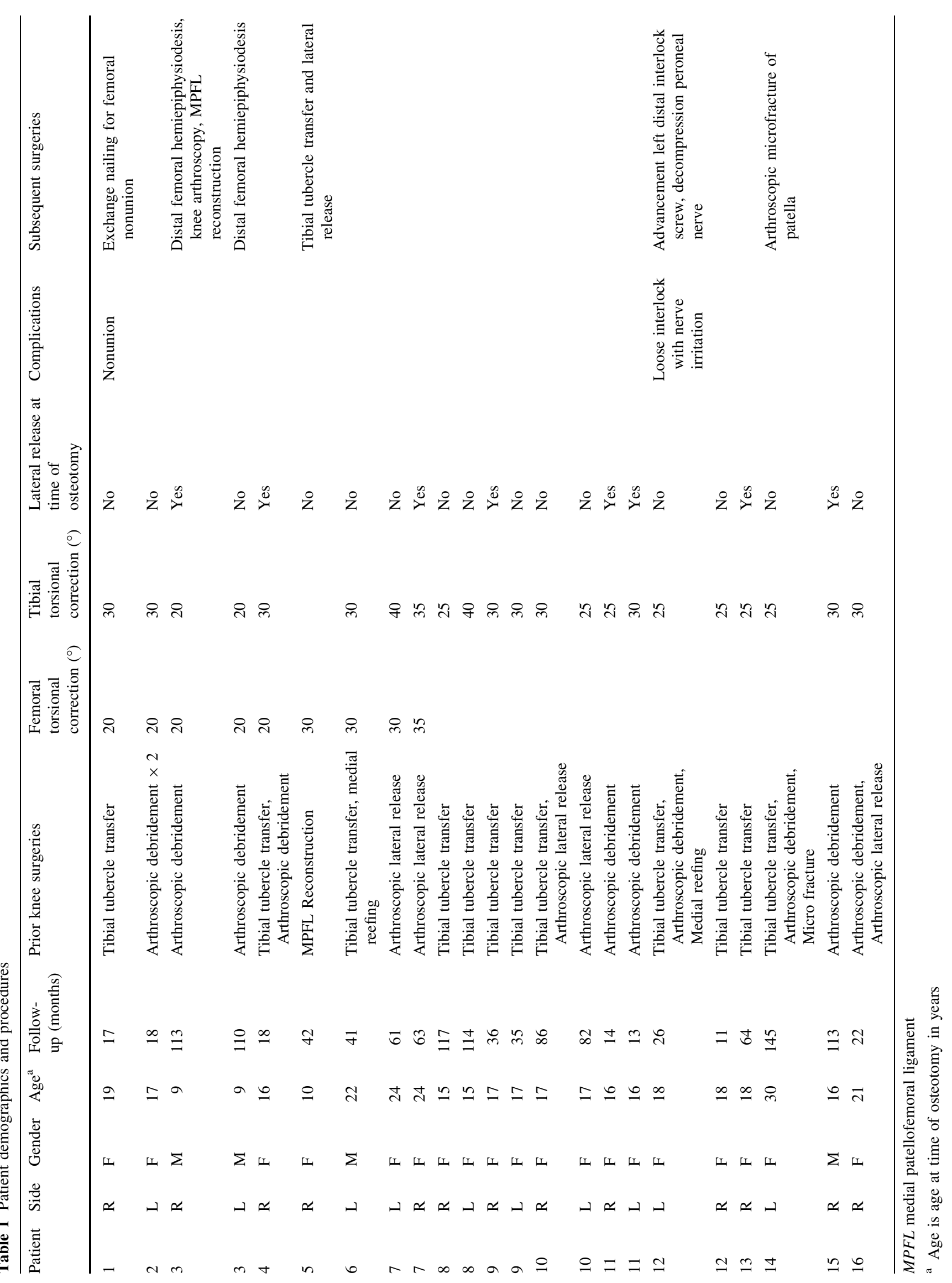


A

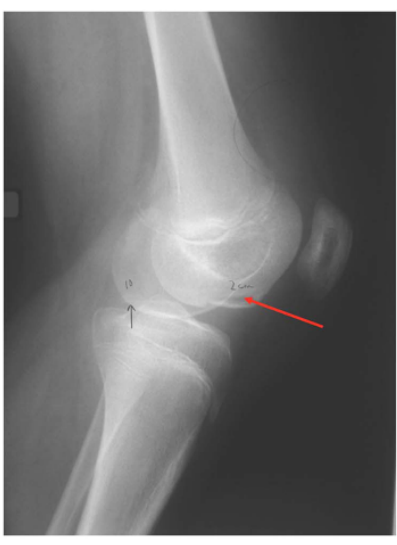

12 y.o. PATELLAR DISLOCATION - SOCCER

C

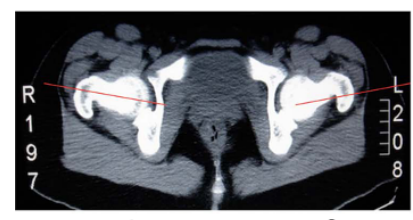

$11^{0}$

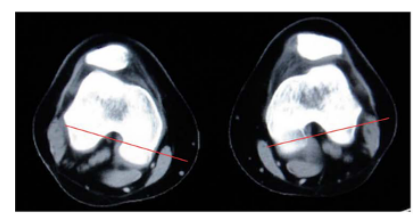

ROTATIONAL “GUNSIGHT” CT SCAN

Fig. 3 a A 12-year-old girl was "tackled" playing soccer and suffered a traumatic dislocation of her left patella. The arrow depicts the osteochondral fracture. b Three years (and four knee operations) later, she continues to have left patellar instability. She is now developing knee symptoms on the right. c Her torsional profile demonstrated $30^{\circ}$ of excessive outward tibial torsion bilaterally. Her

Table 2 Operative details ( $n=23$ rotational surgeries)

\begin{tabular}{lrl}
\hline Operative detail & \multicolumn{1}{c}{ Number $^{\mathrm{a}}$} & Amount of correction $^{\mathrm{b}}$ \\
\hline Femoral osteotomy & $9(39 \%)$ & $25(20-35)$ \\
Tibial osteotomy & $22(96 \%)$ & $29(20-40)$ \\
Both femoral and tibial & $8(35 \%)$ & $54(40-70)^{\mathrm{c}}$ \\
Lateral release & $8(35 \%)$ &
\end{tabular}

${ }^{a}$ Data presented as mean with percentage of total cases in parentheses

${ }^{b}$ Data presented as mean with range in parentheses

c Data presented as mean total combined correction of tibial and femoral osteotomies with range in parentheses

genu valgum, bilateral patellar realignment, and MPFL reconstruction. The second patient required a knee scope debridement and microfracture (Table 1).
B
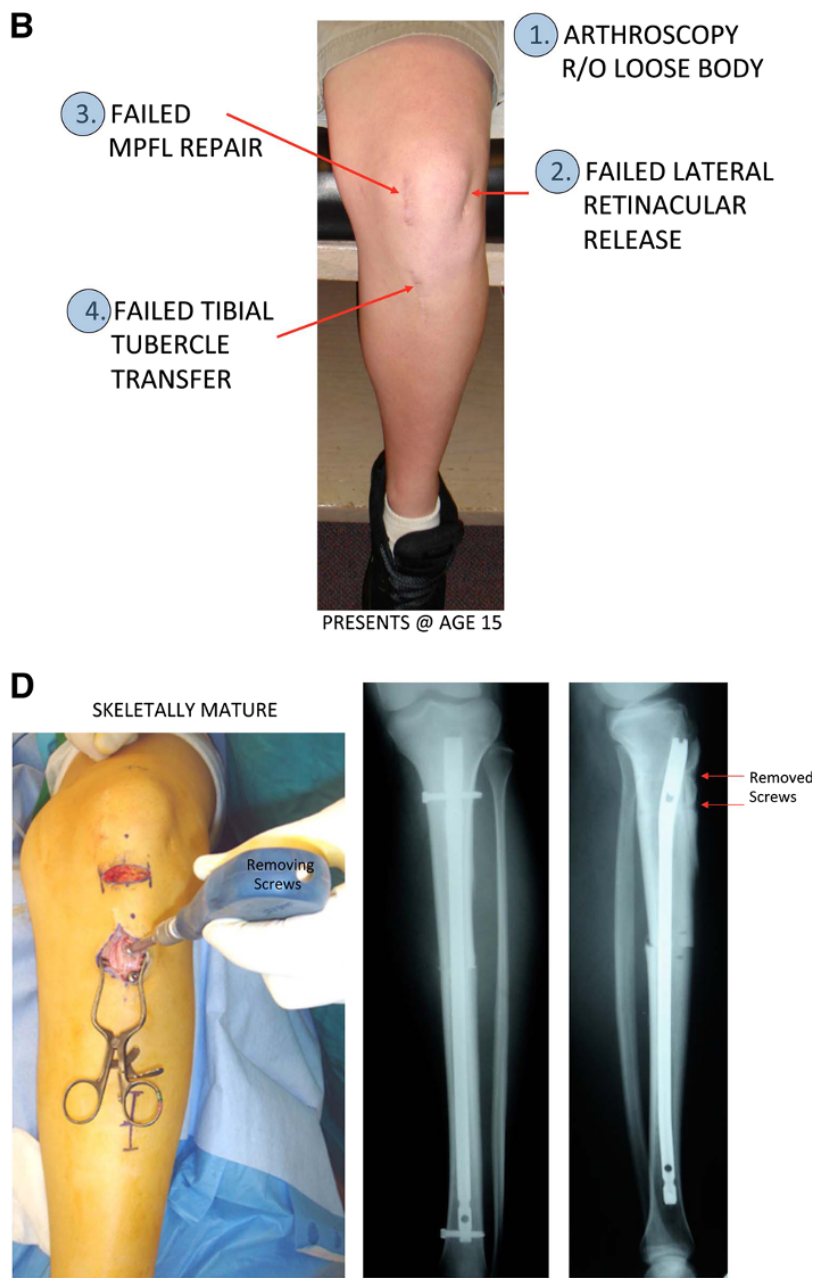

"gunsight" CT scan corroborates the excessive bilateral tibial outward torsion, in the presence of normal femoral version. d At the time of her $30^{\circ}$ rotational tibial osteotomy, the tubercle screws were removed, leaving the transferred tubercle in situ. Two months later, she underwent a rotational osteotomy of her right tibia. At latest follow-up, she has stable patellae and is asymptomatic

\section{Discussion}

Persistent torsional deformities of the femur and/or tibia often go unrecognized in patients with anterior knee pain and/or patellofemoral instability. As the associated acute and chronic symptoms are most often at the knee, there is a tendency for the orthopedic surgeon to focus on that level, without screening for rotational abnormalities of the femur and/or tibia. There is a lingering belief that the physiologic torsional deformities, observed during childhood, are uniformly benign and self-limiting [12]. This belief may result in a failure to appreciate the consequences of persistent and pathological torsion; even Staheli states that "persistent problems are often genetically determined and may only be corrected by osteotomy." Unfortunately, failing to screen for torsional deformities can lead to the detriment of some 
Table 3 Clinical outcome measures $(n=23$ knees with mean follow-up of 59 months)

\begin{tabular}{|c|c|c|c|}
\hline Pain & Preoperative & Postoperative & $p$ value \\
\hline Pain on scale of $1-10^{\mathrm{a}}$ & $8.6(7.9-9.4)$ & $3.3(2.0-4.6)$ & $<0.001$ \\
\hline \multicolumn{3}{|c|}{ Outcomes questionnaire item } & Postoperative response \\
\hline \multicolumn{3}{|c|}{ Do you continue to have knee pain/problems? } & $70 \%$ Yes \\
\hline \multicolumn{3}{|c|}{ Have you had any patellar instability since surgery? } & $43 \%$ Yes \\
\hline \multicolumn{3}{|c|}{ Do you "trust" your knee? } & $57 \%$ Yes \\
\hline \multirow{3}{*}{\multicolumn{3}{|c|}{ Have you changed your lifestyle/activities? }} & \multirow{3}{*}{$\begin{array}{l}65 \% \text { Increased } \\
13 \% \text { Same } \\
22 \% \text { Decreased }\end{array}$} \\
\hline & & & \\
\hline & & & \\
\hline \multicolumn{3}{|c|}{ Have you had knee surgery since your osteotomy/hardware removal? } & $22 \%$ Yes \\
\hline
\end{tabular}

${ }^{\text {a }}$ Data presented as means with $95 \%$ confidence intervals in parentheses management, the possibility that there is a proximate cause of the problem should be kept in mind [1, 2, 8, 16]. In fact, Flandry and Hughston [15] showed a high failure rate for extensor mechanism realignment when the underlying cause of patellofemoral pain was excessive femoral anteversion, external tibial torsion, or both.

A careful clinical assessment is most revealing and is paramount to successful clinical management. When the patient stands with their feet parallel, the patella should be facing forward. The "foot progression angle" should be neutral when walking $[12,17]$. When presenting individually, femoral anteversion is manifest by in-toeing and outward tibial torsion by out-toeing. However, if there is concomitant femoral anteversion and outward tibial torsion, also known as pan genu torsion or "miserable malalignment" [7], the footprint will be neutral and this combined long bone deformity may be concealed to the unwary observer [7]. It is therefore important to have the patient appropriately unclad and note the knee progression angle as well [7, 10, 11].

Malrotation is readily documented by means of assessing the torsional profile in the prone position. Tibial torsion can be documented by measuring the thigh-foot axis (normal $=$ neutral to $15^{\circ}$ outward) [16]. Femoral anteversion may be recognized by identifying, in the prone position, how much inward rotation there is at each hip in extension, compared to outward rotation (normally equal inward and outward rotation $\pm 30^{\circ}$ ) [5, 7]. The findings are not always symmetrical; anecdotally, it is often noted that the affected knee is the one that has more torsion. While plain radiographs do not accurately identify torsion, they are nevertheless helpful. A long-standing anteroposterior radiograph of the legs, with the patellae neutral, will identify any concurrent limb length discrepancy and/or angular deformities. The apparent increase in the femoral neck-shaft angle may be due to increased femoral anteversion. One can observe the presence (or closure) of the physes and measure the limb lengths and mechanical axis. A lateral view of each knee and Merchant [18] or Laurin views [19] of the patella may demonstrate patellar 
malorientation or fragmentation, perhaps as a consequence of long bone torsion.

Significant abnormalities documented on the clinical examination, correlated with the history, may be an indication for advanced imaging. The standard is the "gunsight" CT scan or MRI, employing accepted bony landmarks to define and quantify the degree of malrotation of the femur and/or tibia. While not mandatory, this imaging may help to refine the plans for rotational corrective osteotomies $[2,7-9,11]$. Lee et al showed through a cadaver knee study that a $20^{\circ}$ inward rotational deformity of the femur resulted in increased lateral patellofemoral contact pressures, and a drastic increase in lateral patellofemoral contact pressure resulted when $30^{\circ}$ or more inward rotational deformity was seen [20]. We therefore employ the femoral osteotomies for patients who have greater than $20^{\circ}$ of inward rotational deformity indicated by the preoperative hip range of motion and CT scan. Turner studied rotational profiles in over 1,200 patients and showed consistently that patients with patellofemoral pain and instability had increased tibial outward torsion measuring an average of $24.5^{\circ}$ [16]. We employ inward tibial osteotomies for patients who have greater than $20^{\circ}$ of excess outward rotational deformity [11]. As can be seen in the results from this study, our typical corrective rotations are $25^{\circ}$ in the femur and $30^{\circ}$ in the tibia.

The level and specific technique/fixation of rotational osteotomies remain up to the discretion of the surgeon, which is typically a reflection of training and experience.

With the exception of the immature tibia, we believe that intramedullary fixation offers several advantages [10, 21]. The surgical exposure is minimal, and the comparatively strong load-sharing implants are well tolerated. Immediate mobilization of the joints without cast protection is also appealing to the patient and surgeon alike. In the setting of the skeletally immature tibia, our preferred method of derotation is a distal tibia osteotomy secured with crossing Kirschner wires. We feel that distal tibial osteotomies are simpler, well tolerated, and we have shown that they work well in terms of correcting knee alignment and frontal plane knee moments [11]. In 1994, two separate studies showed improved outcomes for tibial rotational osteotomies when the fibula was left intact [22, 23]. Fibular osteotomy is rarely used in our practice and is reserved for limbs where we cannot easily achieve the desired rotation.

In our experience, patients with anterior knee pain and underlying torsional abnormalities respond very well to derotational corrective osteotomies. In previous work, we evaluated a group of 14 patients with anterior knee pain in 27 limbs with underlying pan genu torsion. These patients all had excellent outcomes with derotational osteotomies of their femurs and tibias [7]. However, none of these patients had prior surgical interventions for their anterior knee pain.
The excellent outcomes seen in our 2004 study provide a stark contrast to the results of the present study comprised entirely of patients who had prior surgeries for their anterior knee symptoms. About $70 \%$ of the current study patients had some continued knee pain, $43 \%$ of these knees had continued patellar instability, and activity level was decreased in $22 \%$ of these patients.

The history of prior failed knee surgery is not a contraindication to corrective osteotomy. In our experience, if a lateral retinacular release has already been undertaken, this may not need to be repeated. If the patellar tendon has been transferred, it may be left in situ, removing the retained screws if an intramedullary device is to be utilized. If the patella remains unstable, revision of patellar alignment should be postponed pending the results of the rotational correction; this may be undertaken at the time of rod removal. Likewise, such draconian measures such as patellectomy or patellofemoral arthroplasty should be postponed unless or until the femoral and/or tibial malrotation have been corrected [14, 24].

There are several limitations to this study. The retrospective nature yields itself to several biases, including selection and recall bias. However, we did attempt to follow these patients prospectively through the use of our outcomes questionnaire. Our outcome measures are inherently limited in that we are not using a validated outcome measure such as the Lysholm knee score [25]. The follow-up on this study was also relatively short-term, limiting conclusions regarding prevention of arthritis, lasting symptom relief, and need for further interventions. A final limitation is that this is a relatively small and heterogeneous group of patients. While we have a prior study of pan genu torsion patients without prior surgery, we feel that this study of patients who had prior failed nontorsional correcting surgery is a useful addition to the literature and a caveat to the knee surgeon.

\section{Conclusion}

In conclusion, we believe that malrotation of the lower limb not only raises the propensity for knee injuries and conditions, but is also a under-recognized etiology in the failure of surgeries for anterior knee pain and/or patellar instability. Further examination of our two series demonstrates that addressing rotational abnormalities in the index surgery yields better clinical outcomes than osteotomies performed after extensor complex transfers or soft-tissue procedures. There are advantages to considering rotational osteotomy early in patients with prominent rotational abnormalities.

Conflict of interest The authors declare that they have no conflict of interest. 
Open Access This article is distributed under the terms of the Creative Commons Attribution License which permits any use, distribution, and reproduction in any medium, provided the original author(s) and the source are credited.

\section{References}

1. Fulkerson JP, Arendt EA (2000) Anterior knee pain in females. Clin Orthop Relat Res 372:69-73

2. Eckhoff DG, Brown AW, Kilcoyne RF, Stamm ER (1997) Knee version associated with anterior knee pain. Clin Orthop Relat Res 339:152-155

3. Fredericson M, Powers CM (2002) Practical management of patellofemoral pain. Clin J Sport Med 12(1):36-38

4. Kolowich PA, Paulos LE, Rosenberg TD, Farnsworth S (1990) Lateral release of the patella: indications and contraindications. Am J Sports Med 18(4):359-365

5. Paulos L, Swanson SC, Stoddard GJ, Barber-Westin S (2009) Surgical correction of limb malalignment for instability of the patella: a comparison of 2 techniques. Am J Sports Med 37(7): $1288-1300$

6. Bellemans J, Cauwenberghs F, Witvrouw E, Brys P, Victor J (1997) Anteromedial tibial tubercle transfer in patients with chronic anterior knee pain and a subluxation-type patellar malalignment. Am J Sports Med 25(3):375-381

7. Bruce WD, Stevens PM (2004) Surgical correction of miserable malalignment syndrome. J Pediatr Orthop 24(4):392-396

8. Eckhoff DG, Montgomery WK, Kilcoyne RF, Stamm ER (1994) Femoral morphometry and anterior knee pain. Clin Orthop Relat Res 302:64-68

9. Stevens PM, Anderson D (2008) Correction of anteversion in skeletally immature patients: percutaneous osteotomy and transtrochanteric intramedullary rod. J Pediatr Orthop 28(3):277-283

10. Stotts AK, Stevens PM (2009) Tibial rotational osteotomy with intramedullary nail fixation. Strategies Trauma Limb Reconstr

11. MacWilliams BA, McMulkin ML, Baird GO, Stevens PM (2010) Distal tibial rotation osteotomies normalize frontal plane knee moments. J Bone Joint Surg Am 92(17):2835-2842

12. Staheli LT (1989) Torsion-treatment indications. Clin Orthop Relat Res 247:61-66
13. Cameron HU (2005) A comedy of errors: the bad knee. J Arthroplast 20(4 Suppl 2):18-22

14. Ramaswamy R, Kosashvili Y, Cameron HU, Cameron JC (2009) Total knee replacement with rotational proximal tibial osteotomy for osteoarthritis with severe external tibial torsion and patellar instability. J Bone Joint Surg 91(11):1466-1471

15. Flandry F, Hughston JC (1995) Complications of extensor mechanism surgery for patellar malalignment. Am J Orthop (Belle Mead NJ) 24(7):534-543

16. Turner MS, Smillie IS (1981) The effect of tibial torsion of the pathology of the knee. J Bone Joint Surg 63-B(3):396-398

17. Staheli LT, Corbett M, Wyss C, King H (1985) Lower-extremity rotational problems in children. Normal values to guide management. J Bone Jt Surg Am 67(1):39-47

18. Merchant AC (2001) Patellofemoral imaging. Clin Orthop Relat Res 389:15-21

19. Laurin CA, Levesque HP, Dussault R, Labelle H, Peides JP (1978) The abnormal lateral patellofemoral angle: a diagnostic roentgenographic sign of recurrent patellar subluxation. J Bone Joint Surg Am 60(1):55-60

20. Lee TQ, Anzel SH, Bennett KA, Pang D, Kim WC (1994) The influence of fixed rotational deformities of the femur on the patellofemoral contact pressures in human cadaver knees. Clin Orthop Relat Res 302:69-74

21. Gordon JE, Pappademos PC, Schoenecker PL, Dobbs MB, Luhmann SJ (2005) Diaphyseal derotational osteotomy with intramedullary fixation for correction of excessive femoral anteversion in children. J Pediatr Orthop 25(4):548-553

22. Manouel M, Johnson LO (1994) The role of fibular osteotomy in rotational osteotomy of the distal tibia. J Pediatr Orthop 14(5):611-614

23. Rattey T, Hyndman J (1994) Rotational osteotomies of the leg: tibia alone versus both tibia and fibula. J Pediatr Orthop 14(5):615-618

24. Price M, Malkani AL, Baker D (2009) Fulkerson procedure for chronic patella component dislocation after total knee arthroplasty. J Arthroplast 24(6):914-917

25. Tegner Y, Lysholm J (1985) Rating systems in the evaluation of knee ligament injuries. Clin Orthop Relat Res 198:43-49 